\title{
The role of magnetic resonance imaging in the diagnosis of breast cancer
}

\author{
Barbara Bobek-Billewicz¹ , Marek K. Jurkowski², 3
}

MR mammography is a highly sensitive (> 98\%) and slightly lower specificity (> 80\%) method of detecting breast cancer. The sensitivity of MR mammography in detecting low or medium grade DCIS is lower than in detecting invasive carcinomas and high grade DCIS. Achieving the high efficacy of MR mammography is only possible with a very good quality MR examination; this however is not always easy to accomplish.

According to EUSOBI 2015 recommendations, the indications for breast MRI are: screening women with a high risk of breast cancer; preoperative staging of newly diagnosed breast cancer; evaluating the response to neoadjuvant chemotherapy; occult primary breast carcinoma (searching for breast cancer in patients with metastases and negative mammography and breast ultrasound); suspected local recurrence whenever needle biopsy proves impossible; assessing breast implants; further characterisation of equivocal lesions found by mammography/breast ultrasound, whenever needle biopsy proves impossible.

The introduction of Digital Breast Tomosynthesis (DBT) and contrast-enhanced spectral mammography (CESM) into daily clinical practice in the recent years has created the need to re-analyse the indications for MR mammography and to develop a new breast cancer diagnostic imaging algorithm.

NOWOTWORY J Oncol 2017; 67, 3: 185-192

Key words: breast cancer, magnetic resonance imaging, breast MRI, MR mammography, occult breast cancer

\section{Introduction}

There are three main imaging modalities used in the diagnosis of breast cancer:

1. X-ray mammography (MMG, 2D MMG). Nowadays ever more frequently performed digitally, however analogue or CR (computed radiography) mammography systems are still in use. Digital mammography enables further diagnostic methods such as DBT (digital breast tomosynthesis) and CESM (contrast-enhanced spectral mammography).

A. DBT eliminates the tissue overlap and allows to assess the breast in $1 \mathrm{~mm}$ slices. It is most commonly undertaken instead of targetted spot compression views, not only allowing further investigation of uncertain or suspicious lesions by the means of 2D MMG, but addi- tionally allowing the assessment of the entire breast. DBT clarifies the nature of most opacities/asymmetric densities observed in single projections and improves the sensitivity and specificity of mammography. In women with diagnosed breast cancer, DBT can be used for the assessment of multifocality / multicentricity. It holds promise for a screening method [1].

B. (Dual energy) contrast-enhanced spectral mammography (CESM) is the earliest of the imaging methods used in diagnosing breast cancer, it is in use since 2011. It is performed after an intravenous injection of an iodine-based contrast agent. Similarly to MR mammography, the contrast enhancement depends on the vascularisation of the lesion [2, 3]. The main indication for CESM

${ }^{1}$ Radiology and Diagnostic Imaging Department, Maria Skłodowska-Curie Memorial Cancer Centre and Institute of Oncology, Branch in Gliwice, Poland

${ }^{2}$ Radiology and Diagnostic Imaging Department, Maria Skłodowska-Curie Memorial Cancer Centre and Institute of Oncology, Branch in Gliwice, Poland

${ }^{3}$ Department of Medical Analytics, Faculty of Medical Science, University of Warmia and Mazury, Olsztyn, Poland 
appear to be equivocal lesions found in mammography or breast ultrasound in patients diagnosed with breast cancer, where additional cancer foci need to be confirmed/excluded (Fig. 1) [4].

2. Ultrasonography. Usually this is classical hand held ultrasound performed by a doctor, which can be supplemented by the assessment of vascularization using the colour Doppler option and by the elastrography. Ultrasound can be performed following an intravenous administration of a contrast agent. A special type of breast ultrasound is the ABUS (Automated Breast Ultrasound), which also allows coronal sections of the breast to be investigated which would be impossible by classical hand held ultrasound examinations; one of its main advantages is repeatability. The examination is performed by a technician/ /radiographer but analysed and reported by a radiologist.

3. MR mammography is a highly sensitivity method in the diagnosis of breast cancer ( $>98 \%$ ) with a slightly lower specificity (> 80\%), and outcomes are influenced by the quality of the examination - this is not always easy to achieve. The sensitivity of MRI in detecting low and intermediate grade DCIS is lower than for the high grade DCIS and invasive cancer [5].

\section{MR mammography}

In order to obtain high quality breast MR, it is necessary to keep to the principles and standards described, inter alia, by the EUSOMA recommendations $[6,7]$.
A. In premenopausal women, the breast MRI should be performed between the 7 th and 14th day of the monthly cycle when the contrast enhancement of the glandular breast tissue is poor and consequently the pathological contrast enhancement of the tumour is more conspicuous and, at the same time, yields less false positive results. If required, this examination can be performed in the third week of the cycle, bearing in mind that the outcome may not be optimal.

B. The MR scanner used for breast MRI examinations should have the minimum magnetic field strength of $1.5 \mathrm{~T}$; a dedicated surface coil for breast imaging should be used.

C. The following sequences should at the very least be obtained: T1-weighted and T2-weighted images, dynamic study following intravenous administration of a Gd-based contrast agent, performed in a T1-weighted high spatial resolution sequence with a suppression of the fatty tissue signal. The dynamic study should consist of 5 repetitions, with each repetition lasting a maximum of 120 seconds (in the currently used systems this should not exceed $90 \mathrm{~s}$ ); the entire study time being approximately 20-30 min.

D. Diffusion weighted MR imaging (DWl/ADC) does not require administering any contrast agent and improve the specificity of the study.

E. The high-dynamic resolution sequence performed in the first 60-90 seconds after intravenous administration

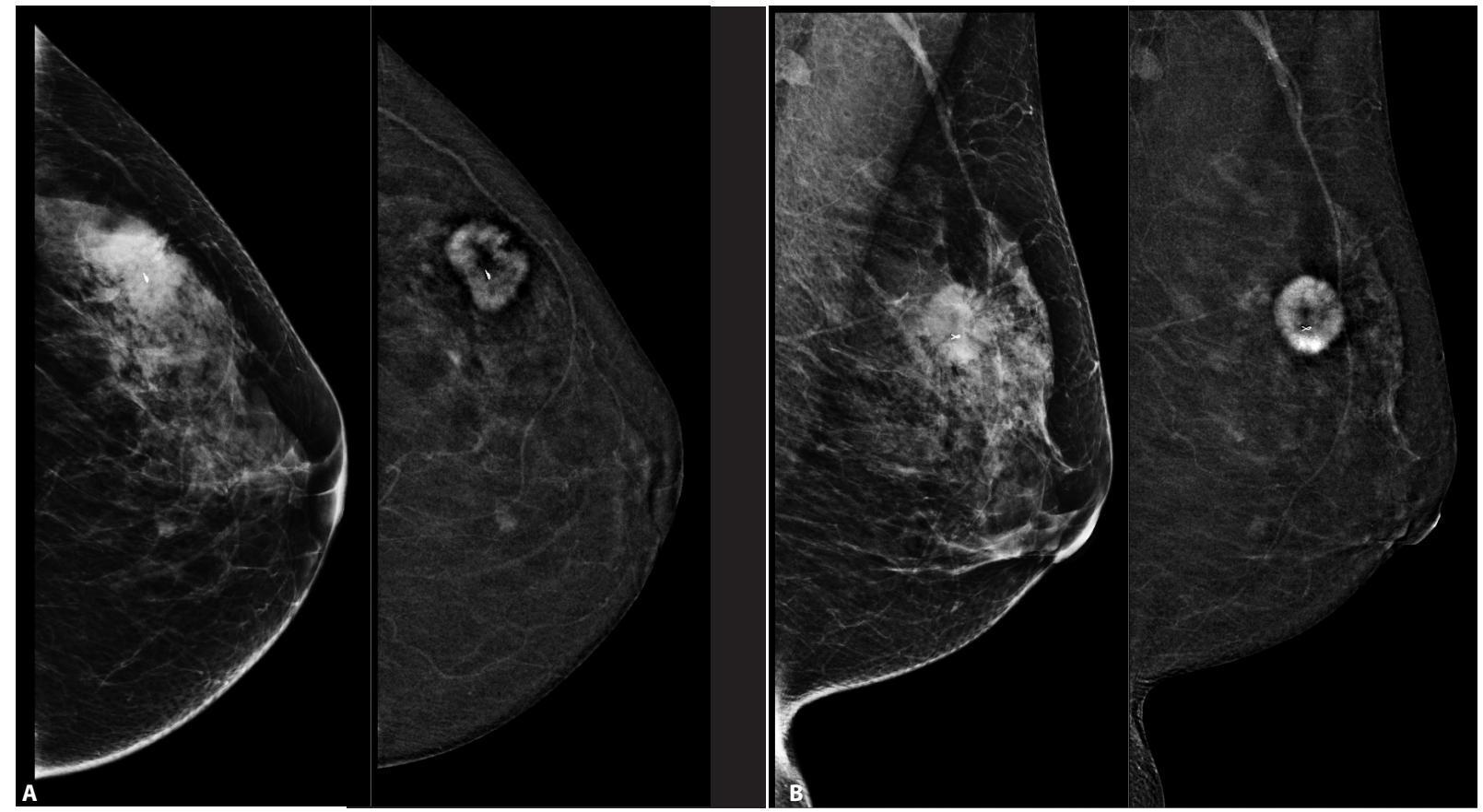

Figure 1.45-year-old woman. Left breast: invasive carcinoma NST, G2, NG2. A. Spectral mammography in CC projection; both images performed after i.v. administartion of a contrast agent. On the left, low energy acquisition like standard mammography. On the right, subtracted image; dual energy mammography with contrast enhancement. B. Spectral mammography in MLO projection; both images performed after i.v. administartion of a contrast agent. On the left, low energy acquisition like standard mammography. On the right, subtracted image; dual energy mammography with contrast enhancement. Contrast-enhancing tumour in the upper outer quadrant of the left breast. Subtracted images showing only contrast-enhancing lesions allow for an improved evaluation of the size and number of cancerous lesions 
of Gd-based contrast agent does not prolong the test time, but it improves the specificity [8].

F. It is vital that the patient is physically placed in such a way that the entire area of interest is covered, ensuring that the patient is comfortable enough to remain motionless for the duration of the test, particularly for the dynamic sequence. Whilst modern MR systems are equipped in motion correction software, this is only possible in certain circumstances.

G. Studies aimed for the evaluation of the implant integrity can be performed without administering an intravenous contrast agent. In cases of silicone implants, dedicated sequences are required for the evaluation of the silicone.

\section{Indications for MR mammography}

These were first comprehensively described in 2010 by the EUSOMA recommendations [6]. From the very beginning, this document has raised controversy and particularly heated discussions concerning the role of the breast MRI in pre-treatment staging of the disease and diagnosing recurrent cancer.

The EUSOBI recommendations presented in 2015 [7] greatly extend the indications for breast MR and include:

A. Screening women with a high risk of breast cancer.

B. Preoperative staging of newly diagnosed breast cancer, Figure 2.

C. Evaluating effects of neoadjuvant chemotherapy.

D. Occult primary breast carcinoma (Fig. 2), (searching for breast cancer in patients with metastases and negative mammography and ultrasound).

E. Suspected local recurrence whenever needle biopsy proves impossible.

F. Evaluating women with breast implants.

G. Further characterisation of equivocal lesions found by mammography / ultrasound, whenever needle biopsy proves impossible.

Indications for breast MRI, that do not raise any significant doubts include:

A. Screening of women running a high risk of breast cancer (BRCA1 or BRCA2 mutation carriers and their relatives), women with other genetic syndromes with increased risk of breast cancer such as the Li-Fraumeni syndrome and Cowden syndrome along with those undergoing chest radiotherapy aged between 10 and 30 years and those having a risk of breast cancer $>20 \%$.

Women with cancer in one breast have an increased risk of developing a second cancer, which may be an indication for performing pre-treatment MR breasts imaging as well as in the post treatment follow up.

B. Assessing the response to neoadjuvant chemotherapy in women with breast cancer.

C. Occult breast cancer.

D. Evaluating implants.
Ad A. MR Mammography is a recognised screening method for women with a high risk of breast cancer [5-7, 9]. Nowadays it is unquestionable that MR imaging should be used for screening women running a high risk of breast cancer; the only debatable point being how this should be performed.

The recommended age for starting MR screening is under discussion, (according to EUSOMA this should be no later than at 30 years), and so are the intervals between screening, (of no longer than 1 year), and the actual screening methodology. Several years ago Christiane Kuhl [10] proposed a shortened MR mammogram protocol as a screening test using a 3 minute acquisition time, comprising performing two successive 3D T1-weighted sequences pre- and post intravenous administration of Gd-based contrast agent. In addition, a 1-2 minute localisation sequence will be needed thus extending the total time to around 5 minutes. The test duration is comparable to that of classical X-ray mammography, but is shorter than sonomammography of both breasts. The authors concluded that such examination, with a 3 minute MR examination and 3-second MIP reconstruction assessment by an expert radiologist being capable of excluding cancer with a NPV of $99.8 \%$. With the time needed to evaluate the entire shortened protocol MRI breasts of less than $30 \mathrm{sec}$, the diagnostic efficacy achieved is comparable to that of the full protocol and additional 18.2 cancers in 1000 were detected [10].

Ad B. Both dynamic imaging (following the administration of an intravenous Gd-based contrast agent) and diffusion-weighted MR imaging are used to assess the response to neoadjuvant chemotherapy (NAC). Even the weak contrast enhancement at the site of the primary tumour post-NAC should be considered as representing residual tumours. Woodhams et al. obtained 96\% efficacy of DWI/ADC (Diffusion-weighted Imaging/Apparent diffusion coefficient) and 89\% DCE (Dynamic contrast-enhanced) efficacy in diagnosing residual tumours [11]. Employing the DWI/ADCs for evaluating residual lesions may be particularly useful in women who, (for example because of renal failure), cannot undergo an intravenous administration of a paramagnetic contrast agent.

Ad C. Occult breast cancer accounts for less than 1\% of breast cancers; in such cases MR imaging is indicated if $X$-ray mammography and breast ultrasound prove negative. Detecting breast cancer allows breast conserving surgery to be adopted instead of mastectomy. For women with breast cancer metastases, there is no clear answer as to whether classical mammography or the DBT and, if appropriate, spectral mammography, should be used before the MRI breasts. For most cases this probably depends on local clinical conditions and the availability of diagnostic imaging methods. As previously mentioned, MR sensitivity for detecting DCIS with low and intermediate grade malignancy is lower than for detecting high-grades of invasive cancer and DCIS (Fig. 3). 

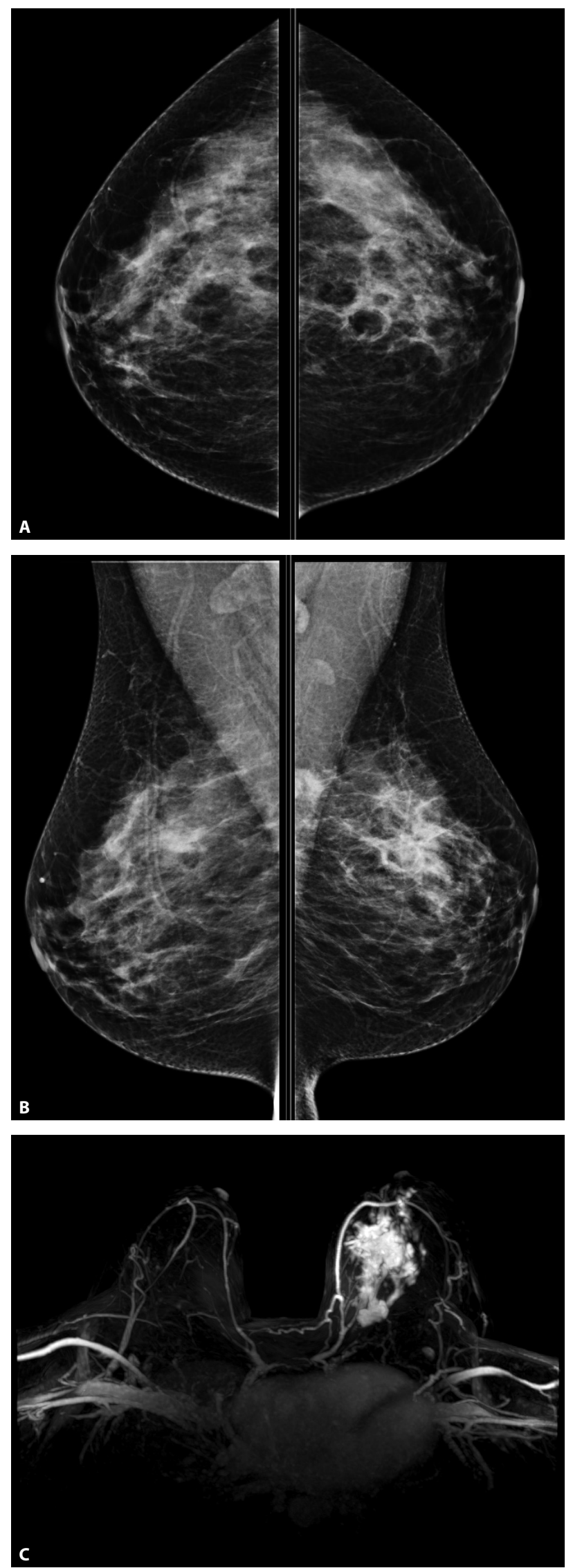

Figure 2. 30-year-old woman. Left breast: invasive carcinoma NST, G2, NG2. A. Mammography — CC projection. B. Mammography — MLO projection; an asymmetric density in the left breast, close to the chest wall, up to about $18 \mathrm{~mm}$ in the largest diameter. C. Breast MRI; MIP reconstruction (maximum intensity projection). An irregular shaped infiltration in the upper outer quadrant and bordering the upper quadrants of the left breast, with the maximum diameter of $66 \times 46 \times 52 \mathrm{~mm}$ 

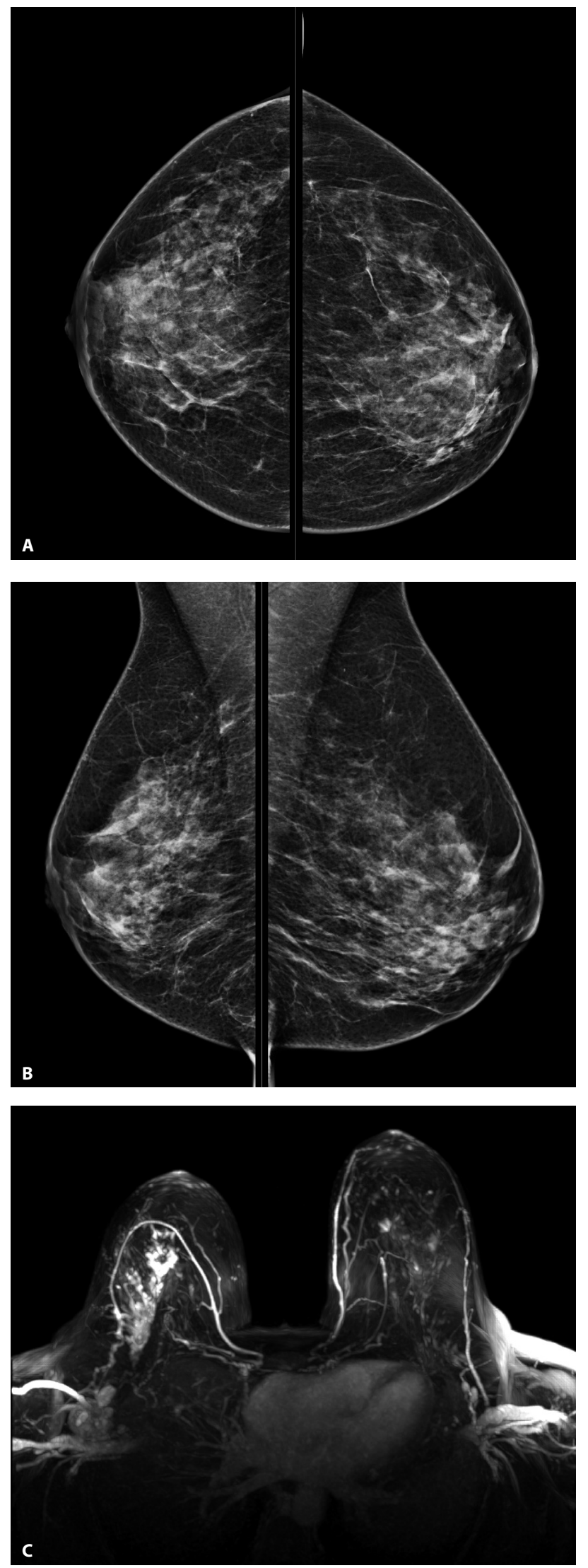

Figure 3.52-year-old woman. Metastases to the right axillary lymph nodes, to the mediastinum and bones. Mammography shows scattered microcalcifications, not forming clusters; DBT did not reveal any suspicious lesions. A. Mammography - CC projection. B. Mammography - MLO projection. C. Breast MRI; MIP reconstruction. An area of abnormal ductal enhancement in the upper outer quadrant of the right breast, measuring up to $75 \times 45 \times 70 \mathrm{~mm}$ in diameter. HP: invasive NST, G3 carcinoma 
Ad D. MR imaging is the gold standard in evaluating the integrity of silicone implants. An MRI scan undertaken solely for the purpose of assessing the implant integrity is performed without administering intravenous contrast agents and is based on the assessment of the sequences dedicated to silicone evaluation. If the purpose is to also detect neoplastic lesions, then complete MR imaging with dynamic imaging after intravenous administration of a paramagnetic contrast agent should be performed as well as the sequences dedicated to the evaluation of the silicone. It is of key importance that implants do not reduce MR sensitivity for detecting breast cancer, including recurrences, and therefore breast MRI appears to be the gold imaging standard in such patients.

The greatest debate concerns the indications for breast MRI prior to starting treatment, local recurrence suspicion and assessing equivocal lesions by the means of mammography and ultrasonography (BIRADS 3, BIRADS 4 A and BIRADS 4B). MR imaging in patients with diagnosed breast cancer generally aims to help select the best treatment option. The first proposed EUSOMA [6] indications recommended that MR imaging should be used prior to treatment in patients with newly diagnosed breast cancer in the following situations:

- invasive lobular carcinoma;

- high risk of breast cancer;

- greater than $1 \mathrm{~cm}$ difference in tumour size when assessed by mammography and ultrasound;

- before PBI (Partial Breast Irradiation).

At present, particularly in the clinical practice, the predominant view is that the MR mammography should be performed to assess the local disease extent prior to starting the treatment, especially in women with a dense and glandular breast structure. This allows the detection of the additional cancer foci both in the breast with the known cancer and the contralateral breast (multi-focality and multi-centicity), as well as to better determine the size of the cancer and thus improve the treatment planning, including the surgical intervention [12]. Paker et al. states that $41 \%$ of surgeons, responding to a 2010 questionnaire issued to members of the American Society of Breast Surgeons, routinely undertook MR mammography for patients with newly diagnosed breast cancer [13].

Undoubtedly MR mammography can detect cancer foci invisible to X-ray mammography and ultrasonography. A meta-analysis by Brennan et al. on 3252 women with unilateral breast cancer found that 131 breast cancer lesions in the contralateral breast were only conspicuousin breast MRI. DCIS comprised 35\% of these lesions [14].

Based on an MR analysis, in women with newly diagnosed breast cancer, Freitas et al. found a high 95\% efficacy of the study, with the NPV of $98 \%$ when excluding occult cancer [15].
A discussion has been raised on the significance for treatment outcome of detecting the cancers invisible by some diagnostic methods and whether all the visible cancer foci should be surgically removed if breast radiotherapy and adjuvant systemic therapy are to be used $[5,16]$. Most assuredly, lesions detected by MR mammography as BIRADS 4 or BIRADS 5 must be microscopically verified before deciding to alter the methods or the scope of treatment. Because most of these lesions are only visible in MR mammography, biopsies must be performed under MR guidance.

MR mammography performed prior to surgical treatment results in increased mastectomy rates [17]. Studies on the effect of preoperative MR mammography on reducing the reoperation rate, when margins prove positive, are inconsistent [18-20] and so are the studies on the effect of preoperative MR imaging on local recurrence rates [21,22].

MR imaging is an effective method for diagnosing local recurrence after the breast conserving treatment. According to the classical indications, it should be performed when the $\mathrm{X}$-ray mammography or breast ultrasound is inconclusive and when a biopsy cannot be undertaken. In clinical practice, MR mammography is often performed as a primary screening test after breast conserving treatment in young women with dense glandular breast. It appears possible that in some cases, MR mammography can be replaced by spectral mammography after an intravenous administration of a contrast agent.

Further characterisation of equivocal lesions, seen in X-ray mammography and breast ultrasound, was not taken into account in the initially proposed indications for performing MR mammography, however in current clinical practice this now usually constitutes the most frequent indication for this study. It should be remembered that MR mammography is much more effective in the assessment of masses, asymmetric densities or architectural distortions rather than in characterising the microcalcification. Suspicious microcalcification seen in mammography should be verified in a biopsy.

A study by Catherine S. Giess et al. from 2016 [23] showed that the main indications for MR mammography should be the situations where there is a doubt as to whether the $X$-ray mammographic findings are clinically relevant or indeed if any lesions actually exist.

Indications for mammography should therefore be asymmetric densities or archtectural distortions visible in only one mammographic projection and the lesions requiring a biopsy, when for whatever reason this proves difficult or impossible to undertake.

Yoo et al. [24] demonstrated the usefulness of MR mammography in assessing the features of well-defined breast lesions. The vast majority of these are benign lesions such as cysts, fibroadenomas or intramammary lymph nodes, but similar ultrasound or mammographic appearances may also 
be produced by cancers and phylloides tumours. This study concluded that MR imaging comprising the analysis of T2-weighted images, of the morphology and of the contrast enhancement kinetics, is useful in differentiating between well-defined benign and malignant lesions.

A meta-analysis by Dorrius Mi et al. [25], assessing BIRADS 3 (probably benign) breast lesions, concluded that MR mammography is useful in the evaluation of BIRADS 3 lesions; NPV of the MR mammography for assessing BIRAD 3 lesions without microcalcification was $100 \%$. According to the authors, no follow up imaging is needed after the MR mammography for the women with X-ray mammography - BIRADS 3 lesions without microcalcification.

An important question is whether MR mammography can reduce the number of unnecessary biopsies of benign lesions, and thus better characterise the BIRADS 4 lesions seen in X-ray mammography and in breast ultrasound.

A study by Strobel et al. on 353 BIRADS 4 breast lesions in 340 women found that $92 \%$ of the biopsies might have been avoided [26]. In order to improve the specificity of the study and therefore reduce the number of false-positive results, a high temporal resolution acquisition, covering the first 60-90 s after i.v. administration of contrast agents, as well as diffusion weighted imaging are used.

A prospective study by Bickelhaupt et al. concluded that diffusion-weighted imaging with background suppression (DWIBS), performed without an administration of an intravenous contrast agent, is helpful in excluding cancer in women with suspecious lesions found in screening X-ray mammography (sensitivity $92 \%$, specificity $94 \%$, NPV 92\%, PPV 93\%) [27].

\section{Conclusions}

1. X-ray mammography supplemented by breast ultrasound scan remains the principal modality in the diagnosis of breast cancer.

2. Tomosynthesis (DBT) reduces the number of equivocal mammographic findings and improves the sensitivity and specificity of mammography.

3. Indications for MR mammography include:

- screening women with a high risk of developing breast cancer;

- preoperative staging of breast lesions in women with known breast cancer;

- evaluating response to neoadjuvant chemotherapy;

- occult primary breast cancer (searching for breast cancer in patients with metastases and negative $\mathrm{X}$-ray mammography and breast ultrasound);

- suspected local recurrence when needle biopsy proves unfeasible;

- further characterisation of equivocal lesions found in $\mathrm{X}$-ray mammography and breast ultrasound;
- evaluating breast implants; importantly, breast implants do not reduce the sensitivity of MR mammography in detecting breast cancer.

4. In some instances, contrast-enhanced spectral mammography may be used instead of MR mammography.

\section{Acknowledgments}

Thanks for dr Tomasz Olejnik (St Mary's Hospital, Newport, Isle of Wight) for helping to prepare the English version.

Conflicts of interest: none declared

Prof. Barbara Bobek-Billewicz, MD, PhD

Radiology and Diagnostic Imaging Department

Maria Skłodowska-Curie Memorial Cancer Centre

and Institute of Oncology, Branch in Gliwice

Wybrzeże Armii Krajowej St. 15

44-101 Gliwice, Poland

e-mail:Barbara.Bobek-Billewicz@io.gliwice.pl

Received \& Accepted: 3 Mar 2017

\section{References}

1. McDonald ES, Oustimov A, Weinstein SP et al. Effectiveness of digital breast tomosynthesis compared with digital mammography: outcomes analysis from 3 years of breast cancer screening. JAMA Oncol 2016; 2 737-743. doi:10.1001/jamaoncol.2015.5536.

2. Jochelson MS, Dershaw DD, Sung JS et al. Bilateral contrast-enhanced dual-energy digital mammography: feasibility and comparison with conventional digital mammography and MR imaging in women with known breast carcinoma. Radiology 2013; 266: 743-751.

3. Dromain C, Thibault F, Muller S et al. Dual-energy contrast-enhanced digital mammography: initial clinical results. Eur Radiol 2011; 21: 565-574.

4. Fallenberg EM, Schmitzberger FF, Amer $\mathrm{H}$ et al. Contrast-enhanced spectral mammography vs. mammography and MRI - clinical performance in a multi-reader evaluation. Eur Radiol 2017; 27: 2752-2764.

5. Morrow M, Waters J, Morris E. MRI for breast cancer screening, diagnosis, and treatment. Lancet 2011; 378: 1804-1811.

6. Sardanelli F, Boetes C, Borisch B et al. Magnetic resonance imaging of the breast: recommendations from the EUSOMA working group. Eur $J$ Cancer 2010; 46: 1296-1316.

7. Mann RM, Balleyguier C, Baltzer PA et al. Breast MRI: EUSOBI recommendations for women's information. Eur Radiol 2015; 25: 3669-3678.

8. Mann RM, Mus RD, van Zelst J et al. A novel approach to contrast-enhanced breast magnetic resonance imaging for screening: high-resolution ultrafast dynamic imaging. Invest Radiol 2014; 49: 579-585.

9. Pijpe A, Andrieu N, Easton DF et al. Exposure to diagnostic radiation and risk of breast cancer among carriers of BRCA $1 / 2$ mutations: retrospective cohort study (GENE-RAD-RISK) BMJ 2012; 345: e5660. doi: 10.1136/bmj.e5660.

10. Kuhl CK, Schrading S, Strobel K et al. Abbreviated breast magnetic resonance imaging (MRI): first postcontrast subtractwed images and maximum intensity projection - a novel approach to breast cancer screening with MRI. J Clin Oncology 2014; 32: 2304-2310.

11. Woodhams $\mathrm{R}$, Kakita $\mathrm{S}$, Hata $\mathrm{H}$ et al. Identification of residual breast carcinoma following neoajuvant chemotherapy: diffusion-weighted imaging-comparison with contrast-enhanced MR imaging and pathologic findings. Radiology 2010; 254: 357-366.

12. Mazilu L, Suceveanu Al, Tomescu D et al. Optimizing the indication for breast-conservative surgery (BCS) in patients with locally-advanced breast cancer. Chirurgia 2013; 108: 478-481.

13. Parker A, Schroen AT, Brenin DR. MRI utilization in newly diagnosed breast cancer: a survey of practicing surgeons. Ann Surg Oncol 2013; 20: $2600-2606$.

14. Brennan ME, Houssami N, Lord S et al. Magnetic resonance imaging screening of the contralateral breast in women with newly diagnosed breast cancer: systematic review and meta-analysis of incremental 
cancer detection and impact on surgical management. J Clin Oncol 2009; 27: 5640-5649.

15. Freitas V, Crystal P, Kulkarni SR et al. The value of breast MRI in highrisk patients with newly diagnosed breast cancer to exclude invasive disease in the contralateral prophylactic mastectomy: Is there a role to choose wisely patients for sentinel node biopsy? Cancer Med 2016; 5: 1031-1036.

16. Pilewskie M, Morrow M. Applications for breast magnetic resonance imaging. Surg Oncol Clin N Am 2014; 23: 431-449.

17. Houssami N, Turner R, Morrow M. Preoperative magnetic resonance imaging in breast cancer: meta-analysis of surgical outcomes. Ann Surg 2013; 257: 249-255.

18. Mann RM, Loo CE, Wobbes T et al. The impact of preoperative breast $\mathrm{MRI}$ on the re-excision rate in invasive lobular carcinoma of the breast. Breast Cancer Res Treat 2010; 119: 415-422.

19. Peters NH, van Esser S, van den Bosch MA et al. Preoperative MRI and surgical management in patients with nonpalpable breast cancer: the MONET - randomised controlled trial. Eur J Cancer 2011; 47: 879-886.

20. Turnbull L, Brown S, Harvey I et al. Comparative effectiveness of MRI in breast cancer (COMICE) trial: a randomised controlled trial. Lancet 2010; 375: 563-571.
21. Fischer $U$, Zachariae $O$, Baum F et al. The influence of preoperative MRI of the breasts on recurrence rate in patients with breast cancer. Eur Radiol 2004; 14: 1725-1731.

22. Solin LJ, Orel SG, Hwang WT et al. Relationship of breast magnetic resonance imaging to outcome after breast-conservation treatment with radiation for women with early-stage invasive breast carcinoma or ductal carcinoma in situ. J Clin Oncol 2008; 26: 386-391.

23. Giess CS, Chikarmane SA, Sippo DA et al. MR imaging for equivocal mammographic findings: help or hindrance? Radiographics 2016; 36: 943-958.

24. Yoo JL, Woo OH, Kim YKet al. Can MR imaging contribute in characterizing well-circumscribed breast carcinomas? Radiopraphics 2010; 30: 1689-1702.

25. Dorrius MD, Pijnappel RM, Jansen-van der Weide MC et al. Breast magnetic resonance imaging as a problem-solving modality in mammographic BI-REDS 3 lessions. Cancer Imaging 2010; 10 Spec No 1A: S54-S58.

26. Strobel K, Schrading S, Hansen NL et al. Assessment of BI-RADS category 4 lesions detected with sceening mammography and screening US. Utility od MR imaging. Radiology 2015; 274: 343-351.

27. Bickelhaupt $\mathrm{S}$, Laun FB, Tesdorff J et al. Fast and noninvasive characterization of suspicious lesions detected at breast cancer X-ray screening: capability of diffusion-weighted MR imaging with MIPs. Radiology 2016; 278: 689-697. 